\title{
ARTICLE
}

\section{COVID-19 in HSCT recipients: a collaborative study of the Brazilian Society of Marrow Transplantation (SBTMO)}

Liane Esteves Daudt (iD ${ }^{1,2,3 凶}$, Mariana Cristina Moraes Corso ${ }^{1}$, Mariana Nassif Kerbauy $\mathbb{D}^{4}$, Luiz Henrique dos Santos de Assis (iD ${ }^{5}$, Ciliana Rechenmacher ${ }^{1,2}$, lago Colturato ${ }^{6}$, Fernanda Rodrigues Barbieri ${ }^{6}$, Vanderson Rocha ${ }^{7,8,9}$, Livia Mariano ${ }^{7}$, Julia Lopes Garcia ${ }^{4}$, Vanessa Esther Cavalcanti Barreto Dantas ${ }^{10}$, Gisele Loth ${ }^{11}$, Vaneuza Araujo Moreira Funke (iD ${ }^{11}$, Polliany Roberta Dorini Pelegrina ${ }^{12}$, Fernando Barroso Duarte ${ }^{13}$, Roberto Luiz da Silva ${ }^{14}$, Marco Aurelio Salvino Araújo ${ }^{5,15}$, Fabianne Altruda de Moraes Costa Carlesse ${ }^{16,17}$, Ana Virginia Lopes de Sousa ${ }^{17}$, Luana Azevedo Maia ${ }^{18}$, Juliana Folloni Fernandes ${ }^{19}$, Celso Arrais Rodrigues ${ }^{20}$, Carmem Bonfim (iD ${ }^{21}$, Leticia Navarro Gordan Ferreira Martins ${ }^{22}$, Rosana Cipolotti ${ }^{23}$, Erick Menezes Xavier ${ }^{8,9}$, Alessandra Araújo Gomes ${ }^{9}$ Hugo Manuel Paz Morales ${ }^{24,25}$, Anderson J. Simioni ${ }^{6}$, Victor Jablonski Soares (D) ${ }^{26}$, Mariana Bohns Michalowski (iD) ${ }^{1,2,27}$, Nelson Hamerschlak (iD) ${ }^{4}$ and Clarisse Martins Machado (D) ${ }^{4,6,28}$

(c) The Author(s), under exclusive licence to Springer Nature Limited 2022

In the COVID-19 scenario, patients undergoing hematopoietic stem cell transplantation (HSCT) infected with SARS-CoV-2 may have an increased risk of death. Through a national multicenter study, we aimed to describe the impact of COVID-19 on the survival of HSCT recipients in Brazil. Eighty-six patients with a confirmed diagnosis of SARS-CoV-2 (92\% by RT-PCR) were included. There were 24 children and 62 adults receiving an autologous $(n=25)$ and allogeneic $(n=61)$ HSCT for malignant $(n=72)$ and non-malignant ( $n=14)$ disorders. Twenty-six patients died, (10 on autologous (38\%) and 16 patients (62\%) on allogeneic group). The estimated overall survival (OS) at day 40 was $69 \%$. Adults had decreased OS compared to children ( $66 \%$ vs $79 \%, p=0.03$ ). The severity of symptoms at the time of diagnosis, ECOG score, laboratory tests (C-reactive protein, urea values) were higher in patients who died $(p<0.05)$. In conclusion, HSCT recipients infected with SARS-CoV-2 have a high mortality rate mainly in adults and patients with critical initial COVID-19 presentation. These findings show the fragility of HSCT recipients with SARS-CoV-2 infection. Therefore, the importance of adherence to preventive measures is evident, in addition to prioritizing the vaccination of family members and the HSCT team.

Bone Marrow Transplantation (2022) 57:453-459; https://doi.org/10.1038/s41409-021-01561-x

\section{INTRODUCTION}

COVID-19 respiratory syndrome is caused by an RNAbetacoronavirus called SARS-CoV-2 and was classified as a Public Health Emergency of International Concern by the World Health Organization (WHO). Until June 29, 2021, there were more than 3.9 million documented deaths worldwide [1, 2].

Although there is a spectrum of clinical manifestations of SARSCoV-2 infection, the majority of patients are asymptomatic or have only mild symptoms of the upper respiratory tract and about $6-14 \%$ evolve with a severe pulmonary syndrome, requiring admission to the intensive care unit (ICU), with respiratory failure, and death [3-6]. Some risk factors for the occurrence of severe cases have been reported. In a retrospective study that evaluated 1590 hospitalized patients, those who died, had, more often, chronic comorbidities, advanced age, dyspneia and laboratory abnormalities [7]. Comorbidities such as hypertension, diabetes, obesity, and coronary heart disease have been reported as risk factors for disease severity in different studies $[3,6,8]$.

Infections are an important cause of morbidity and mortality for hematopoietic stem cell (HSCT) transplant recipients due to

\footnotetext{
${ }^{1}$ Programa de Pós-Graduação em Saúde da Criança e do Adolescente, Departamento de Pediatria, Universidade Federal do Rio Grande do Sul, Porto Alegre, Brazil. ${ }^{2}$ Laboratório de Pediatria Translacional, Serviço de Pesquisa Experimental, Hospital de Clínicas de Porto Alegre, Porto Alegre, Brazil. ${ }^{3}$ Unidade de Hematologia e Transplante de Medula Óssea Pediátrica, Serviço de Hematologia Clínica, Hospital de Clínicas de Porto Alegre, Porto Alegre, Brazil. ${ }^{4}$ Hospital Israelita Albert Einstein, São Paulo, Brazil. ${ }^{5}$ Hospital São Rafael, Salvador, Brazil. ${ }^{6}$ Hospital Amaral Carvalho, Jaú, Brazil. ${ }^{7}$ Service of Hematology, Transfusion and Cell Therapy, and Laboratory of Medical Investigation in Pathogenesis and Directed Therapy in Onco-Immuno-Hematology (LIM-31), Hospital das Clinicas, Faculty of Medicine, Sao Paulo University (FM-USP), São Paulo, Brazil. ${ }^{8}$ Hospital Nove de Julho, São Paulo, Brazil. ${ }^{9}$ Hospital Sírio-Libanês, São Paulo, Brazil. ${ }^{10}$ REAL Hospital Português de Beneficência em Pernambuco, Recife, Brazil. ${ }^{11}$ Hospital de Clínicas da Universidade Federal do Paraná, Curitiba, Brazil. ${ }^{12}$ Hospital Pequeno Príncipe, Curitiba, Brazil. ${ }^{13}$ Hospital Universitário Walter Cantídio, Fortaleza, Brazil. ${ }^{14}$ Instituto Brasileiro de Controle do Câncer, São Paulo, Brazil. ${ }^{15}$ Hospital Universitário Professor Edgar Santos, Universidade Federal da Bahia, Salvador, Brazil. ${ }^{16}$ Universidade Federal de São Paulo, São Paulo, Brazil. ${ }^{17}$ Instituto de Oncologia Pediátrica - GRAACC/UNIFESP, São Paulo, Brazil. ${ }^{18} \mathrm{Hospital}$ Infantil Albert Sabin, Fortaleza, Brazil. ${ }^{19}$ Instituto da Criança do Hospital das Clínicas, Faculdade de Medicina da Universidade de São Paulo, São Paulo, Brazil. ${ }^{20} \mathrm{Hospital} \mathrm{São} \mathrm{Paulo,} \mathrm{Universidade} \mathrm{Federal} \mathrm{de} \mathrm{São} \mathrm{Paulo,} \mathrm{São} \mathrm{Paulo,} \mathrm{Brazil.}{ }^{21}$ Hospital Nossa Senhora das Graças, Curitiba, Brazil. ${ }^{22}$ Hospital Universitário de Londrina, Universidade Estadual de Londrina, Londrina, Brazil. ${ }^{23}$ Centro de Oncologia de Sergipe Dr. Oswaldo Leite, Aracaju, Brazil. ${ }^{24}$ Hospital Erasto Gaertner, Curitiba, Brazil. ${ }^{25} \mathrm{Hospital}$ Oncopediátrico Erastinho, Curitiba, Brazil. ${ }^{26}$ Faculdade de Medicina, Universidade Federal do Rio Grande do Sul, Porto Alegre, Brazil. ${ }^{27}$ Serviço de Oncologia Pediátrica, Unidade de Hematologia Pediátrica, Hospital de Clínicas de Porto Alegre, Porto Alegre, Brazil. ${ }^{28}$ Laboratório de Virologia (LIM 52), Instituto de Medicina Tropical, Faculdade de Medicina da Universidade de São Paulo, São Paulo, Brazil. ${ }^{凶}$ email: Idaudt@hcpa.edu.br
}

Received: 5 May 2021 Revised: 18 November 2021 Accepted: 23 December 2021

Published online: 13 January 2022 
454

chemotherapy effects such as myelosuppression and mucositis. Moreover, in the allogeneic HSCT setting, the occurrence of viral infection is even higher due to immunosuppressive therapy, graftversus-host disease (GVHD) and its treatment. Due to the risk involved with symptomatic SARS-CoV-2 infection in the HSCT scenario, a collaborative effort by Bone Marrow Transplant Societies has prepared guidelines to assist the clinical call decision to protect recipients and donors in this pandemic scenario. Recommended measures include postponing non-urgent transplants, especially in the setting of non-malignant diseases, performing real-time reverse-transcriptase polymerase chain reaction exams (RT-PCR) for SARS-CoV-2 before transplantation to the recipient and donor, cryopreserving the stem cell product before starting the conditioning regimen or having an alternative donor as backup, in addition to strict adherence to infection control protocols during HSCT hospitalization [9-11].

Despite protective recommendations for transplant planning and management, transplant patients remain subject to infection. Few data have been published to date on the clinical manifestations and results of transplant recipients infected with COVID-19, including patients from middle-income countries. In a study conducted at the Memorial Sloan Kettering Cancer Center, 77 patients who were positive for COVID-19 and had previously received allogeneic or autologous HSCT and CD19-directed chimeric antigen receptor T cell (CAR T) had a high rate of hospitalizations (44\%), and $15 \%$ with severe disease died. Secondary infections were formally documented in $12 \%$, and $43 \%$ required supplemental oxygen [12]. In another study Sharma et al. analyzed $318 \mathrm{HSCT}$ recipients diagnosed with COVID-19 with a median time from HSCT to COVID-19 diagnosis of 17 and 23 months for allogeneic and autologous HSCT recipients, respectively. In 45 (14\%) of the cases the disease was classified as severe and 30 days after the diagnosis of COVID-19, the OS was $68 \%$ for allogeneic HSCT recipients and $67 \%$ for autologous HSCT recipients [12].

The present study describes the main clinical findings and complications of COVID-19 in HSCT patients in Brazil. In this national multicenter cross-sectional study, we aimed to identify the variables associated with OS after COVID-19.

\section{METHODS}

\section{Study design, settings and participants}

Patients undergoing autologous or allogeneic HSCT and diagnosed with COVID-19 infection from March 29 to September 1, 2020 admitted to 21 hospitals in five regions of Brazil were included. We considered only patients with positive results in molecular diagnostic tests (RT-PCR) and/or serology (presence of at least one of the immunoglobulins $M$ or $G$ and clinical signs). Patients with suspected COVID-19 disease based only on clinical or radiological findings were excluded.

\section{Variables analyzed}

The data were recorded through Redcap software, and included variables such as: age, Body Mass Index (BMI), diagnostic method, clinical symptoms, performance status at COVID-19 diagnosis, therapeutic measures, and ICU recovery. Children were defined as those under 18 years old. BMI was calculated and nutritional status (thinness, adequate weight, or overweight/obesity) was defined for each child based on age and sex according to the WHO classification [13]. The severity of the clinical presentation at diagnosis was adapted from the classification proposed by Qiu et al [14]. Briefly, mild disease was defined as no oxygen supplemented; moderate and severe, were placed together for any degree of hypoxemia and supplemental oxygen; and critical, mechanical ventilation and or hemodynamic instability. Adult patients were clinically classified by ECOG Performance Status [15].

The primary outcome was overall survival (OS) of 40 days $[9,12]$ after SARS-CoV-2 infection was confirmed.

\section{Statistical analysis}

We use descriptive statistics to analyze patient, transplantation and disease variables and the impact of SARS-CoV-2 infections in our HSCT population.
Continuous variables were assessed for their distribution with the Shapiro-Wilk test and asymmetric distribution were expressed as medians and 25th and 75th percentiles. Qualitative variables were summarized as absolute and relative frequencies. The significance level adopted was 0.05. Fisher's exact test and Pearson's chi-square test were used for categorical variables and the Mann-Whitney $U$ test for quantitative variables. Clinical symptoms and laboratory results were considered after 7 days of evolution. Median values were used to study the effect of associations in a univariable test. Analysis of variance (ANOVA) was used to verify the distribution of data between three or more groups. Log rank tests (Mantel-Cox) were used to compare Kaplan-Meier survival curves between two or more groups. Multivariate analysis comparing significant variables in univariate analysis were performed. The relationship between variables was performed using Multiple Correspondence Analysis (MCA) considering only the cases with all the complete data. In order to select variables for the best fitting model, the reliability test and the explained variance of the criteria of the first two dimensions were used. SPSS ${ }^{\oplus}$ version 18.0 and Winpepi version 11.65 were used for statistical analyses.

\section{RESULTS}

A total of 86 patients (61 allogeneic and 25 autologous HSCT recipients) were included from March 29th to September, 1st 2020. Twenty-four (28\%) were children and adolescents (median 6.5 [1-17] years) and 62 (72\%) were adults (median 44 [18-74] years). $58 \%$ of the patients were male. COVID-19 occurred at a median of 7 [0-216] months after HSCT. The median follow-up time was 43 days (0-161 days). The diagnosis of COVID-19 was confirmed by RT-PCR in 79 (92\%) patients and by serology (lgM/ $\operatorname{lgG})$ in the remaining patients. Among children, 16 (67\%) were asymptomatic (screening according to their own institutional policies) or had mild symptoms, 1 (4\%) was classified as having a moderate/severe condition and $7(29 \%)$ were considered critical. For the 62 adult patients, $36(58 \%)$ were asymptomatic or with mild symptoms, $6(10 \%)$ were moderate or severe and $20(32 \%)$ were critical [16]. Among the 86 patients in this cohort, 61 required hospitalization, 12 of them in ICU. Other relevant clinical data in adult and pediatric HSCT recipients are described in Table 1.

Twenty-one patients (24\%) were included in institutional treatment protocols for COVID-19 and $2(2 \%)$ in a clinical trial. The remaining patients underwent other treatments that included antivirals for 24\%, where 19 (22\%) were treated with Oseltamivir and $2(2 \%)$ received Ganciclovir. No patient received the drug Remdesivir despite its approval and use in other countries during the period of observation. $43(50 \%)$ patients were treated with antibiotics (azithromycin, amoxicillin, cefepime). Corticosteroids were administered to 24 patients, prophylactic heparin to 22 and full anticoagulation to 2 . Medicines such as hydroxychloroquine (14\%); tocilizumab (1\%); immunoglobulin (1\%) and treatment with convalescent plasma (2\%) were also used. Twenty-six patients required intensive care ( 13 days of hospitalization on average), 12 were discharged and 22 of them died. The clinical evolution of the patients can be seen in Figs. 1 and 2.

The mortality in this cohort was $30 \%$ with a total of 26 deaths. Eighteen patients died due to COVID-19. In the remaining 8 patients, the causes of death were 3 fungal infections, 2 bacterial sepsis, 1 sinusoidal obstruction syndrome, 1 relapse of underlying malignancy and 1 GVHD. The overall COVID-19 lethality rate was $21 \%$ (15\% and $36 \%$ in allogeneic and autologous HSCT recipients, respectively).

Overall survival (OS) estimated at day 40 was $69 \%$. Patients whose initial presentation was considered critical had decreased OS when compared to patients with less severe symptoms (Critical $31 \%$ vs Others $70 \% ; p<0.001 ; \mathrm{IC}=95 \%$ ) (Fig. $3 \mathrm{~A}$ ). Children and adolescents showed an increased OS compared to adults ( $81 \%$ vs $66 \%, p=0.034$ ) (Fig. 3B). In addition, boys had decreased survival rates when compared to girls ( $62 \%$ vs $100 \% ; p=0.049$ ) (Fig. 3 C). In adult patients, gender was not associated with survival. Regarding 
Table 1. Characteristics of hematopoietic stem cell transplantation patients with COVID-19 diagnosis.

\begin{tabular}{|c|c|c|c|c|}
\hline \multirow[t]{2}{*}{ Characteristic } & \multicolumn{4}{|l|}{ COVID-19 (\%) } \\
\hline & $\begin{array}{l}\text { Total } \\
(N=86)\end{array}$ & Children $(N=24)$ & $\begin{array}{l}\text { Adults } \\
(N=62)\end{array}$ & $p$ value $^{a}$ \\
\hline $\operatorname{Sex}(M / F)$ & $50 / 36(1.39: 1)$ & $14 / 10(1.4: 1)$ & $36 / 26(1.38: 1)$ & 1000 \\
\hline \multicolumn{5}{|l|}{ Symptoms related to COVID-19 } \\
\hline Fever & $20(23 \%)$ & $5(21 \%)$ & $15(24 \%)$ & 0.786 \\
\hline Lower respiratory tract symptoms & $19(22 \%)$ & $4(17 \%)$ & $15(24 \%)$ & 0.569 \\
\hline Headache & $6(7 \%)$ & $2(8 \%)$ & $4(7 \%)$ & 0.670 \\
\hline Ageusia/Anosmia & $4(5 \%)$ & $1(4 \%)$ & $3(5 \%)$ & 1000 \\
\hline Diarrhea & $14(16 \%)$ & $1(4 \%)$ & $13(21 \%)$ & 0.062 \\
\hline Asymptomatic & $10(12 \%)$ & $8(33 \%)$ & $2(3 \%)$ & 0.001 \\
\hline Allogeneic related & $30(35 \%)$ & $7(29 \%)$ & $23(37 \%)$ & $0.006^{b}$ \\
\hline Haploidentical & $17(20 \%)$ & $9(38 \%)$ & $8(13 \%)$ & \\
\hline Allogeneic unrelated & $14(16 \%)$ & $6(25 \%)$ & $8(13 \%)$ & \\
\hline Autologous & $25(29 \%)$ & $2(8 \%)$ & $23(37 \%)$ & \\
\hline \multicolumn{5}{|l|}{ Comorbidities } \\
\hline Chronic Kidney Failure & $1(1 \%)$ & $0(0 \%)$ & $1(2 \%)$ & 1000 \\
\hline Diabetes (type 2) & $8(9 \%)$ & $0(0 \%)$ & $8(13 \%)$ & 0.099 \\
\hline Heart disease & $4(5 \%)$ & $1(4 \%)$ & $3(5 \%)$ & 1000 \\
\hline Systemic arterial hypertension & $12(14 \%)$ & $2(8 \%)$ & $10(16 \%)$ & 0.496 \\
\hline
\end{tabular}

${ }^{a} p$ value refers to the comparison between children and adults.

${ }^{\mathrm{b}}$ The $p$ values related to two or more lines refer to analyzes performed using contingency tables. Underlying diseases included: malignant: germ tumor, multiple myeloma, myelodysplastic syndromes, Hodgkin lymphoma, non-Hodgkin lymphoma, chronic myeloid leukemia, acute myeloid leukemia, acute lymphoblastic leukemia; non-malignant: primary immunodeficiency disease, Fanconi anemia, hemoglobinopathies, and aplastic anemia.

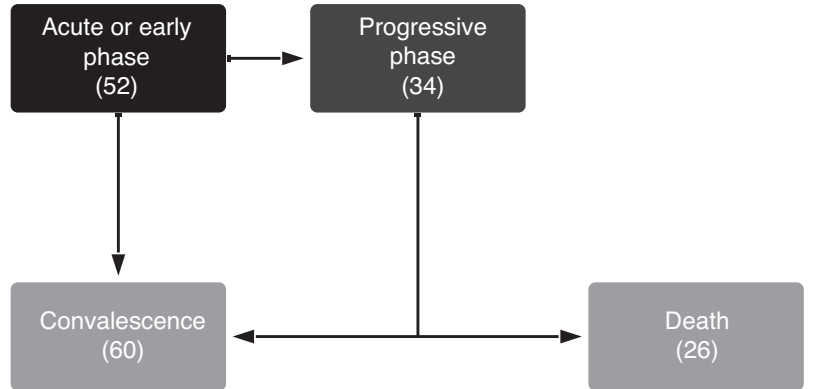

Fig. 1 Clinical evolutions of Hematopoietic Stem Cell Transplantations patients with COVID-19 diagnosis. The parentheses represent the number of patients in each stage of disease evolution. Acute or early, first 7 day from diagnosis; progressive, means maintenance or progression of clinical symptoms after 7 days of diagnosis.

ECOG Performance Status, patients with higher scores (3 e 4) presented worse OS ( $14 \%$ vs $76 \%, p=0.003$ ) (Fig. 3D).

$\mathrm{BMI}, \mathrm{ABO}$ blood typing, type of transplant (allogeneic vs autologous), conditioning regimens or presence of comorbidities were not associated with survival. Laboratory tests were also analyzed at diagnosis and after 7 days hospital admission. The values of C-reactive protein (CRP) and urea at diagnosis were statistically higher in patients who died. Seven days after diagnosis, practically all the laboratory values evaluated were worse for patients who died than for those who survived (Table 2).

Through multivariate analysis, we evaluated the variables severity (mild, moderate/severe, critical vs asymptomatic), age (groups of adults between $41-60$ years and $>60$ years vs $<41$ years), HSCT (autologous vs allogeneic), and ECOG (score $(0,1,2)$ vs $(3,4)$ ). The variables age and HSCT were not significant in this analysis despite being significant in univariate test. However, we observed a difference between the two categories of ECOG $(p=0.01)$ and between patients with mild symptoms vs asymptomatic $(p=0.008)$ (other classifications of severity were not significant in this analysis).

The MCA model represented $75 \%$ of explained variance, and reliability analysis measured by Cronbach's alpha $0.706(95 \% \mathrm{Cl}$ $0.499,0.820$ ) was adequate for exploratory purposes. A cluster of patients classified as severe and critical at clinical presentation and with urea higher than $50 \mathrm{mg} / \mathrm{dL}$ was observed in deceased patients. Instead, creatinine $\leq 2 \mathrm{mg} / \mathrm{dL}$, urea lower than $50 \mathrm{mg} / \mathrm{dL}$, and a clinical mild or asymptomatic presentation are associated with OS. Figure 4 also shows a trend toward allogeneic recipients and with an ECOG score of $0-2$ for better survival. 


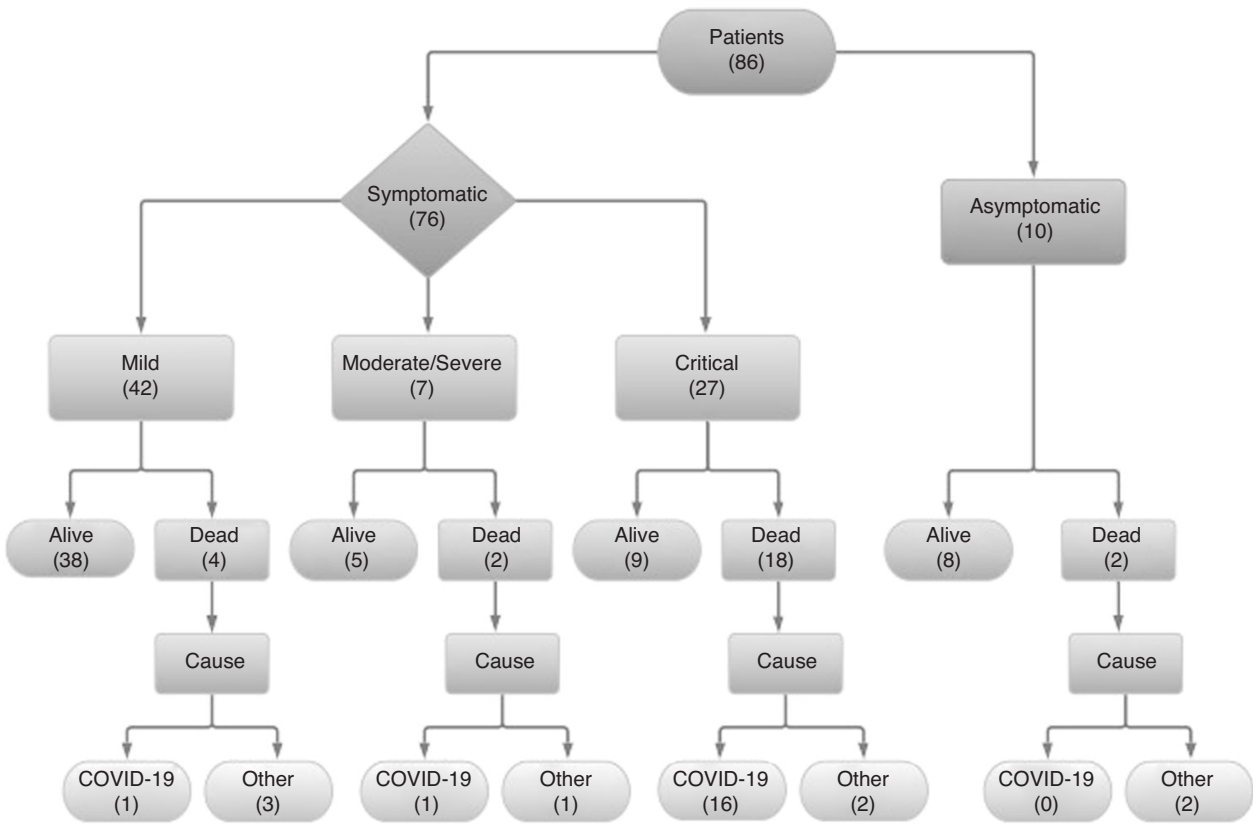

Fig. 2 Flowchart of COVID-19 clinical progression according to initial symptoms (number of patients). Mild disease: no oxygen supplemented; moderate and severe, any degree of hypoxemia and supplemental oxygen; and critical, mechanical ventilation and or hemodynamic instability.

a

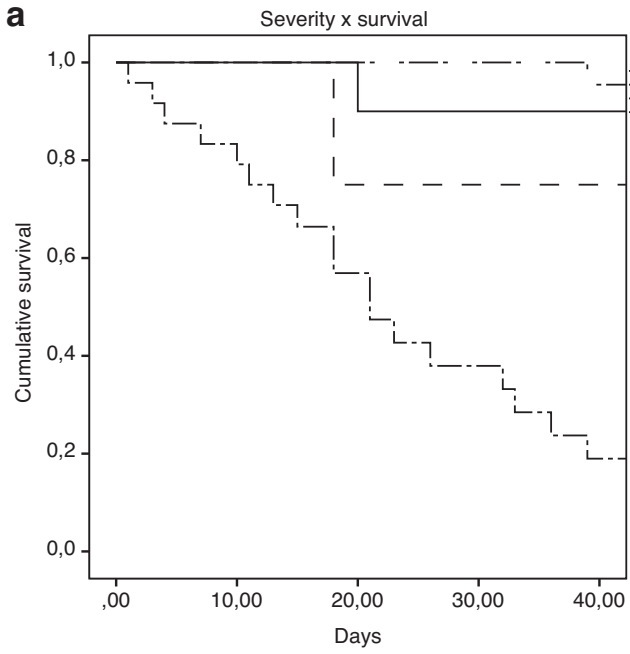

C

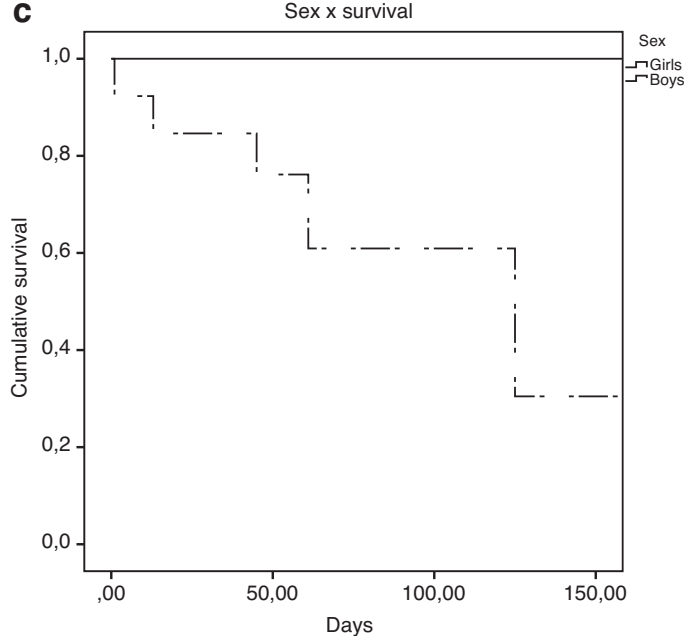

b Age $x$ survival

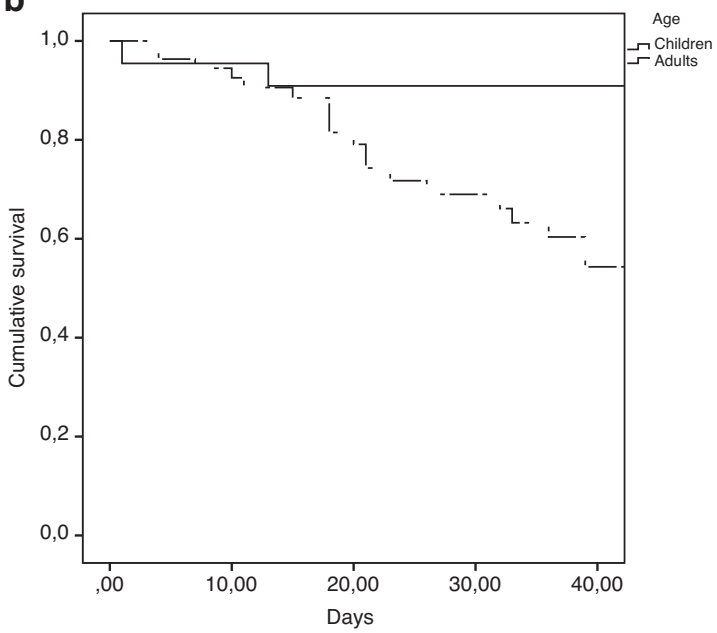

d

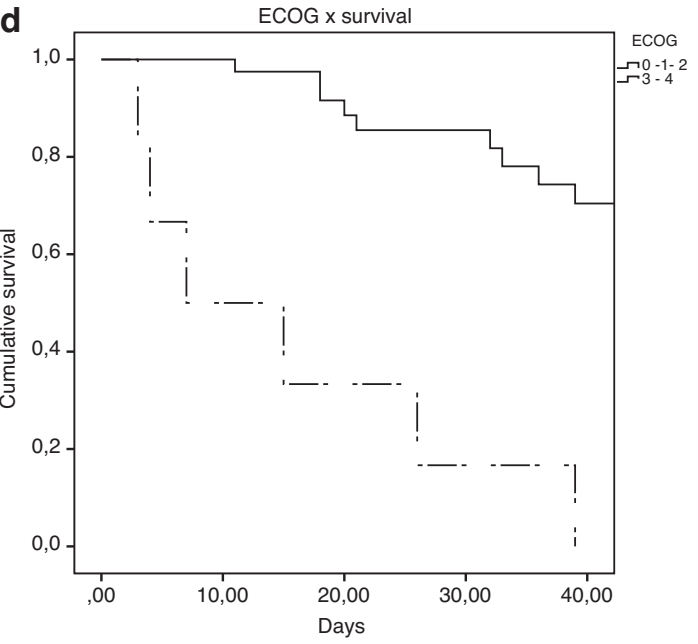

Fig. 3 Cumulative survival after COVID-19:A: Severity of the condition at diagnosis $(p<0.001)$; B: Age (Children $\times$ Adults) ( $p=0.034)$; C: Sex (pediatrics) $(p=0.049) ; \mathbf{D}$ : ECOG (adult patients) $(p=0.003)$. 
Table 2. Laboratory characteristics at diagnosis and at 7 days after the diagnosis of COVID-19 Hematopoietic Stem Cell recipients.

\begin{tabular}{|c|c|c|c|c|c|c|c|c|}
\hline \multirow[t]{3}{*}{ Characteristics } & \multicolumn{4}{|c|}{ Laboratory results at diagnosis } & \multicolumn{4}{|c|}{ Laboratory results 7 days after diagnosis } \\
\hline & \multicolumn{4}{|l|}{ Outcome } & \multicolumn{4}{|l|}{ Outcome } \\
\hline & & Deceased & Alive & $p$ value & & Deceased & Alive & $p$ value \\
\hline \multicolumn{9}{|c|}{ Hemoglobin $(g / d L)$} \\
\hline$n$ & 74 & 26 & 48 & 0.058 & 56 & 19 & 37 & $0.004^{\mathrm{a}}$ \\
\hline Median & 10.2 & 9.1 & 10.65 & & 9.7 & 8.5 & 10 & \\
\hline Min/Max & $5.4 / 15.4$ & $6.5 / 14.5$ & $5.4 / 15.4$ & & $6.8 / 16.4$ & $6.8 / 14.3$ & $7 / 16.4$ & \\
\hline \multicolumn{9}{|l|}{ Neutrophils (mL) } \\
\hline $\mathrm{n}$ & 72 & 25 & 47 & 0.401 & 55 & 18 & 37 & 0.590 \\
\hline Median & 2588.5 & 3850 & 2310 & & 1753 & 1989.5 & 1753 & \\
\hline Min/Max & $0 / 13,637$ & $0 / 13,637$ & $0 / 8152$ & & $0 / 9048$ & $0 / 5000$ & $0 / 9048$ & \\
\hline \multicolumn{9}{|c|}{ Lymphocyte (mg/ L) } \\
\hline $\mathrm{n}$ & 70 & 24 & 46 & 0.608 & 65 & 22 & 43 & $0.042^{\mathrm{a}}$ \\
\hline Median & 890 & 700 & 1061 & & 690 & 452 & 858 & \\
\hline Min/Max & $0 / 7424$ & $0 / 3334$ & $0 / 7424$ & & $0 / 10,210$ & $0 / 2615$ & $0 / 10,210$ & \\
\hline \multicolumn{9}{|l|}{ Platelet $(\mathrm{mL})$} \\
\hline$n$ & 73 & 26 & 47 & 0.080 & 55 & 18 & 37 & $0.007^{\mathrm{a}}$ \\
\hline Median & 84,000 & 83,300 & 88,000 & & 67,000 & 37,000 & 89,000 & \\
\hline Min/Max & $291 / 565,000$ & $9000 / 242,000$ & $291 / 565,000$ & $10,000 / 474,000$ & $10,000 / 136,000$ & $11,000 / 474,000$ & & \\
\hline \multicolumn{9}{|c|}{ Creatinine $(\mathrm{mg} / \mathrm{dL})$} \\
\hline $\mathrm{n}$ & 72 & 25 & 47 & 0.435 & 65 & 23 & 42 & $0.007^{\mathrm{a}}$ \\
\hline Median & 0.84 & 0.91 & 0.80 & & 0.86 & 0.98 & 0.71 & \\
\hline Min/Max & $0.1 / 17$ & $0.10 / 17$ & $0.10 / 2.01$ & & $0.1 / 56$ & $0.1 / 56$ & $0.13 / 2.78$ & \\
\hline \multicolumn{9}{|l|}{ Urea (mg/dL) } \\
\hline$n$ & 66 & 25 & 41 & $0.003^{a}$ & 62 & 21 & 41 & $0.001^{\mathrm{a}}$ \\
\hline Median & 31 & 43 & 27 & & 34 & 59 & 30 & \\
\hline Min/Max & $5 / 138$ & $5 / 138$ & $10 / 76$ & & $7 / 140$ & $11 / 140$ & Jul-74 & \\
\hline \multicolumn{9}{|l|}{ D-dimer ( $\mathrm{mg} / \mathrm{L})$} \\
\hline$n$ & 56 & 20 & 36 & 0.054 & 29 & 9 & 20 & $0.038^{\mathrm{a}}$ \\
\hline Median & 614 & 909.5 & 536 & & 655 & 890 & 421.5 & \\
\hline $\operatorname{Min} / \operatorname{Max}$ & $0.1 / 17,350$ & $1.12 / 17,350$ & $0.1 / 6576$ & & $0.28 / 7010$ & $2.4 / 7010$ & $0.28 / 1790$ & \\
\hline \multicolumn{9}{|c|}{ Fibrinogen $(\mathrm{mg} / \mathrm{dL}$ ) } \\
\hline $\mathrm{n}$ & 24 & 12 & 12 & 0.623 & 21 & 11 & 10 & $0.011^{\mathrm{a}}$ \\
\hline Median & 431 & 460.5 & 429 & & 448 & 513 & 349 & \\
\hline Min/Max & $5.3 / 791$ & $5.3 / 791$ & $60 / 774$ & & $80 / 780$ & $275 / 660$ & $80 / 780$ & \\
\hline \multicolumn{9}{|c|}{ C-reactive protein $(\mathrm{ng} / \mathrm{mL}$ ) } \\
\hline $\mathrm{n}$ & 67 & 24 & 43 & $0.028^{\mathrm{a}}$ & 49 & 20 & 29 & $0.031^{\mathrm{a}}$ \\
\hline Median & 24 & 59.55 & 15 & & 35.29 & 57.5 & 14.3 & \\
\hline Min/Max & $0.13 / 339,000$ & $1.21 / 248$ & $0.13 / 339,000$ & $0.15 / 243,000$ & $3.9 / 355.2$ & $0.15 / 243,000$ & & \\
\hline
\end{tabular}

${ }^{\text {a }}$ Statistically significant difference $(p<0.05)$.

\section{DISCUSSION}

Viral infections are among the main causes of morbidity and mortality in HSCT populations. For this reason, there is great concern regarding immunosuppressed patients infected with SARS-CoV-2. After the conditioning regimen (mainly in allogeneic settings) these populations become especially vulnerable due to delayed immune reconstitution. This picture is potentially aggravated by the use of immunosuppressive therapy and other complications, such as GVHD and transplantation-related morbidities. Community-acquired viral respiratory infections can affect at least $50 \%$ of transplanted patients, with varied outcomes [16]. Historically, the Coronavirus family (with regard to SARS-CoV-1 and MERS-(OV) has not been associated with more severe disease in severely immunosuppressed populations (transplants, chemotherapy or immunosuppressants). The risk of contracting this disease was comparable to the general population [17]. There is little data of COVID-19 in HSCT recipients and our study is the first to describe the presentation of COVID-19 in the HSCT population on a national scale in Latin America.

Regarding the clinical symptoms, the most frequent respiratory symptoms were cough and signs of respiratory dysfunction. The asymptomatic condition observed in children was significantly higher than that diagnosed in adults $(p<0.001)$. These results are very similar to those previously described by Felsenstein et al., where children were also more frequently asymptomatic. According to them, one of the possible explanations for mild presentation in childhood may be the frequent contact to seasonal coronaviruses, as a result, the presence of antibodies with crossreactivity and co-elimination with other viral infections. The authors also hypothesized that vaccinations and associated heterologous immune responses, and a more diverse memory $\mathrm{T}$ cell may provide advantages over the elderly population [18].

Our results are similar in comparison with other case series recently published. In the present study, the estimated OS at day 40 was $69.8 \%$ (children $79 \%$ vs adults $66 \%$ ). Considering the type of HSCT, we observed an OS of $72.9 \%$ among patients undergoing allogeneic transplantation and $60 \%$ in the autologous transplantation group. The outcome in autologous HSCT recipients was slightly inferior to what was previously described in the study conducted by the European Society for Blood and Marrow Transplantation (EBMT), where $\sim 500$ transplant patients in 22 countries were included. At 6 weeks, mortality rate was 19\% and $24 \%$ in autologous and allogeneic HSCT, respectively [9]. Likewise, in a study by Akshay Sharma et al., with 318 patients, from the 


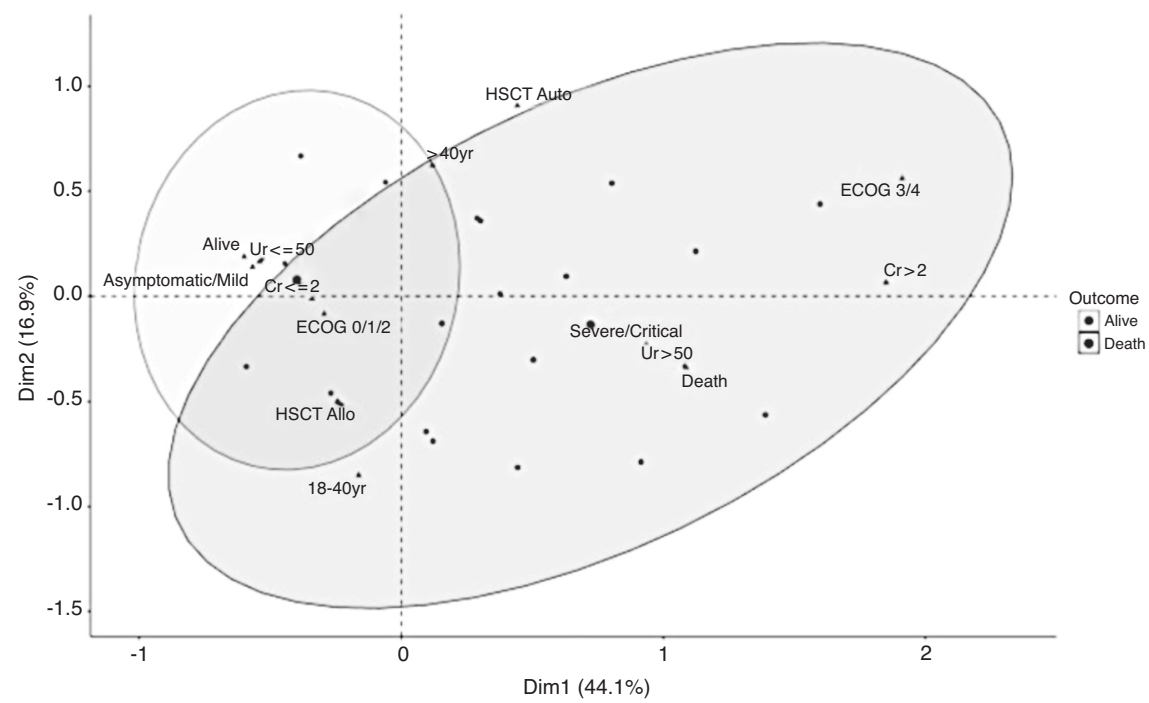

Fig. 4 Multivariate Correspondence Analysis between the main factors related to survival. MCA Multiple Correspondence Analysis, Dim Dimension, $C r$ serum creatinine $\mathrm{mg} / \mathrm{dL}$, Ur serum urea $\mathrm{mg} / \mathrm{dL}$, yr years old, HSCT Allo Allogeneic hematopoietic stem cell transplantation, HSCT Auto Autologous hematopoietic stem cell transplantation, ECOG: Eastern Cooperative Oncology Group Performance Status, Severity of clinical presentation: asymptomatic/mild and severe/critical (see text).

Center of International Blood and Marrow Transplant Research, the OS observed 30 days after the diagnosis of COVID-19 was $68 \%$ for allogeneic and $67 \%$ for autologous. The latter study also showed an association between the diagnosis of COVID-19 and mortality in the following 12 months in allogeneic HSCT recipients [12]. We did not observe any significant association between mortality and time of occurrence of COVID-19 after HSCT during a follow-up of 7 months. In our study, we were not able to individualize differences to explain this finding, however, it is worth emphasizing that mean age tended to be higher in patients in the autologous group and possibly associated with other comorbidities, although no statistical difference was found. In this study, we also did not explore differences in measures of protection or care between the evaluated groups.

According to data provided by the Ministry of Health, on June 30,2021 , Brazil accumulated the number of deaths related to COVID-19 above 515,900 and a fatality rate of 2.8\% [19]. Although Brazil has a Unified Health System (SUS) that guarantees assistance to all, the rapid and progressive increase in cases of COVID-19, especially in low-income populations, put a lot of pressure on hospital care, leading to the saturation of hospital and ICU beds, which certainly impacted final outcomes. Such premise was reinforced by the results obtained by Baqui et al., were 11,321 Brazilian patients with COVID-19 admitted to hospitals with severe acute conditions were analyzed. Higher mortality rates were observed in the northern region of the country in comparison to the central-south region ( $65 \%$ vs $41 \%)$, the so-called "regional effect". The authors also demonstrated that comorbidities were more common in Brazilians admitted to hospitals in the northern region. The study theorizes that the regional effect is driven by an increase in the burden of comorbidities in regions with lower levels of socioeconomic development and that the ethnic effect is possibly related to differences in the susceptibility of COVID-19 and access to health care [20].

We also show that the initial clinical presentation was decisive in the prognosis. As noted in the EBMT record, patients with impaired performance status at COVID-19 diagnosis, and worsening inflammatory tests and kidney function had worse prognosis. Due to the absence of specific therapies, the early identification of patients with higher risk of unfavorable evolution and the prompt introduction of supportive therapy can determine the evolution of the infection in this specific group of patients.
Few data exist in the literature on characteristics of patients after HSCT with COVID-19. Garnica et al. described a total of 11 patients, all with hematological neoplasms, but only two after the transplant. Of these, 5 died. $45 \%$ had neutropenia, $73 \%$ had lymphocyte count below $1000 / \mathrm{mm}^{3}$ [21]. Shah et al., when analyzing 77 patients with SARS-CoV-2 who were recipients of cellular therapy found that neutropenia, comorbidities and presence of infiltrates on initial imaging were associated with more severe disease and death (25 of 77) [22]. In our study, neutropenia was present in $26 \%$ of the subjects, and lymphopenia in 53\%, (data available for 72 and 70 patients, respectively) Variables such as urea, CRP, fibrinogen, d-dimers, creatinine, lymphocytes, and hemoglobin (evaluated 7 days after hospitalization) showed significant difference between survival groups. The qualitative MCA (Fig. 4) analysis carried out also showed that variables such as urea and creatine, as well as the severity of the clinical presentation, seem to be related to the outcome. Also showed a tendency for allogeneic recipient patients to survive longer than the group of autologous recipients, although this was not significant in other analyzes carried out in this study. Although the analysis is limited due to the small sample size, these findings are relevant as they point to indicators that may be related to worse outcomes.

Our study has some limitations. First, Brazil is a country of continental dimensions and significant regional differences. Thus, our group of patients is heterogeneous including children and adults, allogeneic and autologous HSCT for malignant and nonmalignant diseases. Second, since patients' follow-up was short, it was not possible to assess long-term consequences of COVID-19 in these patients. Finally, although some laboratory results may indicate a worse prognosis, biomarkers could not be robustly evaluated due to the lack of data. Despite this, it is a representative sample of post-transplant patients treated in 21 centers in our country and the largest series of cases published so far in a middle-income country.

Even with the protective measures generally applied to these patients and many changes imposed by the pandemic in the BMT centers, infection by SARS-CoV-2 represents a great challenge for the team and the patients. Despite advances in the understanding of pathophysiology and therapeutic measures, prevention remains the main measure to minimize its impact and should be strongly recommended [23]. Understanding the delayed sequelae in 
surviving patients, the impact of new mutations and worldwide mass vaccination on the persistence of circulating SARS-COV-2, and the efficacy of current COVID-19 vaccines in HSCT recipients will be the challenges for years to come.

In summary, this is the first multicenter retrospective study that evaluated the impact of SARS-CoV-2 on survival of HSCT recipients in Latin America. Long-term outcomes should be evaluated in the future, such as the impact of COVID-19 sequelae and the influence of infections on the quality of life of these patients.

\section{REFERENCES}

1. Huang C, Wang $Y$, Li X, Ren L, Zhao Jet, Hu Y, et al. Clinical features of patients infected with 2019 novel coronavirus in Wuhan, China. Lancet. 2020;395:497-506.

2. World Health Organization - WHO. Coronavirus 2021 https://www.who.int/ emergencies/diseases/novel-coronavirus-2019 Accessed 29 June 2021.

3. Gandhi RT, Lynch JB, del Rio C. Mild or Moderate Covid-19. N Engl J Med. 2020;383:1757-66.

4. Zhou F, Yu T, Du R, Du R, Fan G, Liu Y, et al. Clinical course and risk factors for mortality of adult inpatients with COVID-19 in Wuhan, China: a retrospective cohort study. Lancet. 2020;395:1054-62.

5. Guan W, Ni Z, Hu Y, Liang W, Ou C, He J, et al. Clinical Characteristics of Coronavirus Disease 2019 in China. N Engl J Med. 2020;382:1708-20.

6. Richardson S, Hirsch JS, Narasimhan M, Crawford JM, McGinn T, Davidson K, et al. Presenting Characteristics, Comorbidities, and Outcomes Among 5700 Patients Hospitalized With COVID-19 in the New York City Area. JAMA. 2020;323:2052-9.

7. Chen R, Liang W, Jiang M, Guan W, Zhan C, Wang T, et al. Risk Factors of Fatal Outcome in Hospitalized Subjects With Coronavirus Disease 2019 From a Nationwide Analysis in China. Chest. 2020;158:97-105.

8. Xie J, Tong Z, Guan X, Du B, Qiu H. Clinical Characteristics of Patients Who Died of Coronavirus Disease 2019 in China. JAMA Netw Open. 2020;3:e205619.

9. Ljungman P, Mikulska M, de la Camara R, Basak G, Chabannon C, Corbacioglu S, et al. The challenge of COVID-19 and hematopoietic cell transplantation; EBMT recommendations for management of hematopoietic cell transplant recipients, their donors, and patients undergoing CAR T-cell therapy. Bone Marrow Transpl. 2020;55:2071.

10. Algwaiz G, Aljurf M, Koh M, Horowitz M, Ljungman P, Wiesdorf D, et al. Real-World Issues and Potential Solutions in Hematopoietic Cell Transplantation during the COVID-19 Pandemic: Perspectives from the Worldwide Network for Blood and Marrow Transplantation and Center for International Blood and Marrow Transplant Research Health Services and International Studies Committee. Biol Blood Marrow Transpl. 2020;26:2181-9.

11. Machado CM. COVID-19 and hematopoietic stem cell transplantation: recommendations from the Brazilian Society of Bone Marrow Transplantation (SBTMO). Jbmtct. 2020;1:67-72.

12. Sharma A, Bhatt NS, St Martin A, Abid MB, Bloomquist J, Chemaly RF, et al. Clinical characteristics and outcomes of COVID-19 in haematopoietic stem-cell transplantation recipients: an observational cohort study. Lancet Haematol [Internet]. 2021;3026:1-9.

13. De Onis, M., Onyango, A. W., Borghi, E., Garza, C. \& Yang, H. Comparison of the World Health Organization (WHO) Child Growth Standards and the National Center for Health Statistics/WHO international growth reference: Implications for child health programmes. Public Health Nutr. 2006;9:942-7.

14. Qiu H, Wu J, Hong L, Luo Y, Song Q, Chen D. Clinical and epidemiological features of 36 children with coronavirus disease 2019 (COVID-19) in Zhejiang, China: an observational cohort study. Lancet Infect Dis. 2020;20:689-96.

15. Oken MM, Creech RH, Tormey DC, Horton J, Davis TE, McFadden ET, et al. Toxicity and response criteria of the Eastern Cooperative Oncology Group. Am J Clin Oncol. 1982;5:649-55.

16. Samaha R, Kattan J. Hematopoietic stem cell transplantation dilemma during the COVID-19 era. Futur Oncol. 2020;16:1569-73.

17. D'Antiga L. Coronaviruses and immunosuppressed patients: the facts during the third epidemic. Liver Transpl. 2020;26:832-4.
18. Felsenstein S, Hedrich CM. SARS-CoV-2 infections in children and young people. Clin Immunol. 2020;220:108588.

19. Painel Coronavírus. Coronavírus Brasil. https://covid.saude.gov.br/. Published 2021. Accessed June 29, 2021

20. Baqui P, Bica I, Marra V, Ercole A, van der Schaar M. Ethnic and regional variations in hospital mortality from COVID-19 in Brazil: a cross-sectional observational study. Lancet Glob Heal [Internet]. 2020;8:e1018-26.

21. Garnica M, Valetim MR, Furtado P, Moreira MC, Bigni R, Vinhas S, et al. COVID-19 in hematology: data from a hematologic and transplant unit. Hematol Transfus Cell Ther Oct. Dec 2020;42:293-9.

22. Shah GL, DeWolf S, Lee YJ, Tamari R, Dahi PB, Lavery JA, et al. Favorable outcomes of COVID-19 in recipients of hematopoietic cell transplantation. J Clin Investig. 2020;130:6656-67.

23. Duarte FB, Moura ATG, Hallack Neto AE, Seber A, Vigorito AC, Lourenço ALG, et al. Comparative Analysis of the Data on the Influence of the Sars-Cov-2 Pandemic on Bone Marrow Transplantation and the Protocols Adopted in Brazil Between May and June 2020. Jbmtct 2020;2:63-8.

\section{ACKNOWLEDGEMENTS}

We would like to thank all the researchers from the centers that are participating in the registration, as well as the Brazilian Association of Hematology and Hemotherapy $(\mathrm{ABHH})$ and the Brazilian Society of Bone Marrow Transplantation (SBTMO) for all the support for the study.

\section{AUTHOR CONTRIBUTIONS}

LED and MCMC conceptualized and designed the study, drafted the initial paper, and reviewed and revised the paper. IC, FRB, LM, JLG, VECBD, GL, VAMF, PDP, FBD, RLS, MASA, FAMCC, AVLdeS, LAM, JFF, CAR, CMSB, LNGFM, RC, EMX AAG, HM, AJS collected data, carried out the initial analyses, and reviewed and revised the paper. MNK, LHA, CR, VJS, VR, MBM, NH and CMM conceptualized and designed the study, coordinated and supervised data collection, and critically reviewed the paper for important intellectual content. All authors approved the final paper as submitted and agree to be accountable for all aspects of the work.

\section{FUNDING}

The research was supported by FIPE/HCPA (Fundo de Incentivo à Pesquisa e Eventos do Hospital de Clínicas de Porto Alegre). Role of Funder/Sponsor: Financial support with office supplies and digital database maintenance.

\section{COMPETING INTERESTS}

The authors declare no competing interests.

\section{ETHICS APPROVAL AND CONSENT TO PARTICIPATE}

Ethical approval to conduct this study has been granted by the Ethics Committee of Hospital de Clínicas de Porto Alegre (HCPA). Responsible for minors' signed informed consents in duplicate, keeping a copy for themselves.

\section{ADDITIONAL INFORMATION}

Correspondence and requests for materials should be addressed to Liane Esteves Daudt.

Reprints and permission information is available at http://www.nature.com/ reprints

Publisher's note Springer Nature remains neutral with regard to jurisdictional claims in published maps and institutional affiliations. 